\title{
Coroas Totais de Resina Composta Direta: Relato de Casos Clínicos
}

\section{Direct Composite Resin Total Crown: Case Report}

Guilherme Anziliero Arossi ${ }^{1}$ Leandro Azambuja Reichert $^{2}$, Adair Luiz Stefanello Busato ${ }^{3}$

\section{Abstract}

The constant evolution of surgical techniques and restorative materials has allowed impressive advances in dental reconstructions, allowing endodontically treated teeth with extensive coronary destruction to be reconstructed in a single session. The clinical application of composite resins, especially in posterior teeth has been widely accepted by professionals and patients due to the aesthetic issue and the resistance that the resin materials developed in the last 20 years. As for professionals, following the correct protocol is essential to improve the aesthetics and longevity of the restoration. This paper aims, through clinical case reports, to describe techniques for restoring largely destroyed anterior and posterior teeth using composite resins to build up crowns attached to direct fiberglass prefabricated pins. The cases reported here are an alternative procedure, low cost and rapid development, which could meet the needs of the patient, being a good choice of dental oral rehabilitation.

Key-words: permanent dental restoration, dental crowns, composite resins.

\section{Resumo}

A evolução constante das técnicas operatórias e dos materiais restauradores tem permitido avanços impressionantes nas reconstruções dentárias, permitindo que dentes tratados endodonticamente com amplas destruições coronárias seja reconstruídos em sessão única. A aplicabilidade clínica das resinas compostas, principalmente em dentes posteriores, vem sendo bastante aceita pelos profissionais e pacientes devido à questão estética e à resistência que os materiais resinosos desenvolveram nesses últimos 20 anos. Já para os profissionais, seguir o correto protocolo clínico é fundamental para aperfeiçoar a estética e a longevidade da restauração. Este trabalho tem como objetivo, através do relato de casos clínicos, descrever técnicas para restauração de dentes anteriores e posteriores amplamente destruídos utilizando resinas compostas na confecção de coroas totais diretas associadas a pinos de fibra de vidro pré-fabricados. Os casos aqui relatados constituem-se num procedimento alternativo, de baixo custo, e rápida elaboração, que conseguiu suprir as necessidades do paciente, mostrando ser uma boa opção de tratamento reabilitador.

Palavras-chave: restauração dentária permanente, coroas dentárias, resinas compostas.

\author{
${ }^{1}$ Professor de clínica integral do curso de Odontologia (ULBRA) - Torres \\ ${ }^{2}$ Professor de clínica integral do curso de Odontologia da (ULBRA) - Canoas \\ ${ }^{3}$ Coordenador do curso de Odontologia da (ULBRA) - Canoas
}

Correspondência: Guilherme Anziliero Arossi

Endereço: Rua Universitária, 1900 - CEP 95560-000, Parque de Balonismo, Torres - RS, Brasil

Fone: (51) 9718-8336 - (51) 3626-2000 - Coordenação do Curso de Odontologia

E-mail: guilhermeclinica@gmail.com; guilherme.arossi@ulbra.br

Data de Submissão:

Data de Aceite:

\section{Introdução}

A mudança de paradigma estabelecida pelo desenvolvimento de ciências afins - como o advento da adesão, um maior conhecimento em cariologia e o desenvolvimento tecnológico de equipamentos que se consolidaram na última década - resultou em um novo perfil operatório por parte dos profissionais da odontologia e um novo perfil dentário na população (BUSATO et al., 2001; THYLSTROOP, FEJERSKOV, 2001; BUSATO; HERNANDEZ; MACEDO, 2002). A queda do CPO-D e CPO-S vêm transformando a demanda dos serviços odontológicos, de uma odontologia cirúrgicorestauradora baseada na intervenção, para um enfoque preservador, baseado na conservação, na manutenção, na estética e na saúde do paciente (KRIEGER; BEZERRA, 1997; PINTO, 2000).

Restaurações de resina composta em dentes posteriores passaram a se tornar um protocolo corriqueiro na maioria dos consultórios e clínicas odontológicas, gerando uma nova forma de pensar e fazer Odontologia, baseada na máxima prevenção, máxima preservação e mínima restauração (BUSATO; HERNANDEZ; MACEDO, 2002). O que uma vez era o novo, com intervenções conservadoras que objetivavam a manutenção máxima de tecido dental sadio e restaurações estéticas adesivas, passou a ser o atual.

Dessa maneira, cada vez mais as pessoas mantêm por mais tempo os dentes na boca, e por mais tempo esses dentes são expostos aos desafios físicos, mecânicos, químicos e bacterianos, acarretando uma demanda por restaurações dentárias individuais de diferentes tamanhos e extensões. Com a evolução dos materiais restauradores, ampliaram-se suas indicações de uso no dia-a-dia clinico. Um exemplo são as resinas compostas, que tiveram suas propriedades mecânicas e estéticas aprimoradas (SANTOS JUNIOR, 2000; CONCEIÇÃO; MASOTTI; HIRATA, 2005) nos últimos anos permitindo seu uso até mesmo em grandes reconstruções de dentes posteriores, o que tradicionalmente era realizado somente com restaurações metálicas e/ou cerâmicas (DELIPERI, 2008). Os avanços da odontologia restauradora, através da adesividade em esmalte e dentina (BUONOCORE, 1955; NAKABAYASHI; KOJIMA; MASUHARA, 1982), permitiram o emprego de restaurações adesivas associadas aos pinos intracanais nos casos de grande perda de estrutura dentária, sendo considerados uma boa alternativa na reconstrução de dentes fraturados (CHEUNG, 2005; DELIPERI, 2008).

A perda de resistência que ocorre em dentes com pequeno remanescente dental é melhor restabelecida com restaurações indiretas, contudo, as diretas conseguem atingir níveis satisfatórios de 
desempenho para utilização clinica (DALPINO et al., 2002; CHRISTENSEN, 2007). Em dentes desvitalizados, estas restaurações diretas são associadas a pinos intracanais préfabricados, que transmitem menor força lateralmente às paredes da raiz, diminuindo as chances de fratura (CHEUNG, 2005). Reforçando o potencial restaurador dos materiais resinosos, há evidência que compósitos têm uma melhor capacidade de absorção de forças quando comparados às porcelanas (BRUNTON et al. 1999) e que restaurar dentes com recobrimento de cúspides aumenta a durabilidade da restauração (OPDAM et al., 2008).

Diversos relatos são encontrados mostrando a aplicabilidade clinica de grandes reconstruções diretas e indiretas em dentes anteriores e posteriores, para resolução estética de dentes com amelogênese imperfeita, além de indicados para a síndrome do dente trincado, onde a melhor opção de tratamento é a coroa total, uma vez que em restaurações diretas se observou um grande índice de fratura das cúspides. Também relatam vantagens como preservação do remanescente dental, facilidade na técnica, diversidade de cores e efeitos, e os consertos que se tornarem necessários são facilmente conseguidos sem despesas laboratoriais, com total controle do cirurgião-dentista (TAMBELLINI; RAZABONI, 1995; CAVALCANTI; SANTOS; GUEDES-PINTO, 2002; CHRISTENSEN, 2004; ALBUQUERQUE et al., 2005; MANFIO et al., 2006; MARTURELLI et al., 2007).

Sabe-se que a cerâmica é um material com ótimas propriedades funcionais e estéticas (SPOHR; CONCEIÇÃO, 2005) e tem sua longevidade comprovada (CHRISTENSEN, 2005), porém, apresenta desvantagens como maior tempo clínico necessário a sua confecção, além de elevado custo para paciente e profissional (MANFIO et al., 2006). Por esse motivo, aumentou o interesse em trabalhos que mostrem a possibilidade de uso de materiais resinosos de forma direta nas mesmas situações. Preocupado com a longevidade desses procedimentos, Deliperi (2008) acompanhou por 3 anos uma restauração extensa de resina composta direta em um molar endodonticamente tratado. Observou que não houve pigmentação marginal, cárie secundária e nem lascas. Reforça as qualidades dos pinos de fibra de vidro e afirma que restaurações amplas em resina composta são uma boa alternativa de tratamento, desde que se domine a técnica adesiva.

Este trabalho relata dois casos clínicos de coroas totais de resina composta em dentes anteriores e posteriores confeccionados pela técnica direta.

\section{Relato de Casos}

\section{Relato de caso clínico 1}

Paciente 53 anos, sexo feminino, leucoderma, compareceu à clínica odontológica descontente com a restauração do dente 44 que se apresentava escurecida e sem anatomia, além de não apresentar o ponto de contato distal (Figura 1).

O dente possuía tratamento endodôntico considerado satisfatório clinico e radiograficamente, e necessidade de restaurar grande porção da estrutura dental original (Figura 2). Iniciou-se com o acesso ao tratamento endodôntico e desobturação parcial do canal com ponta diamantada 1091 e 1014 (KGSorensen, Barueri, SP, Brasil), e broca Largo $n^{\circ} 2$ (Microdent, São Paulo, SP, Brasil) removendo $2 / 3$ do material obturador $(\approx 12 \mathrm{~mm}$ ), e cimentação de pino de fibra de vidro (Superpost Lightcore $n^{01}$ - Superdont, Rio de Janeiro, RJ, Brasil) com cimento ionômero de vidro trial Vitremer (3MESPE, St Paul, MN, USA); para aumentar a área adesiva da restauração e distribuir melhor os esforços mastigatórios da futura restauração (Figuras 3 e 4). Uma vez concluída a desobturação parcial e profilaxia do canal com álcool, inseriu-se o cimento com seringa Centrix (DFL, Rio de Janeiro, RJ, Brasil) e broca Lentulo (Dentsply Maillefer, Petrópolis, RJ, Brasil); e fotopolimerizou-se por 60 segundos juntamente com o pino de fibra devidamente posicionado (Optilight LD III GNATUS, Ribeirão Preto, SP, Brasil).

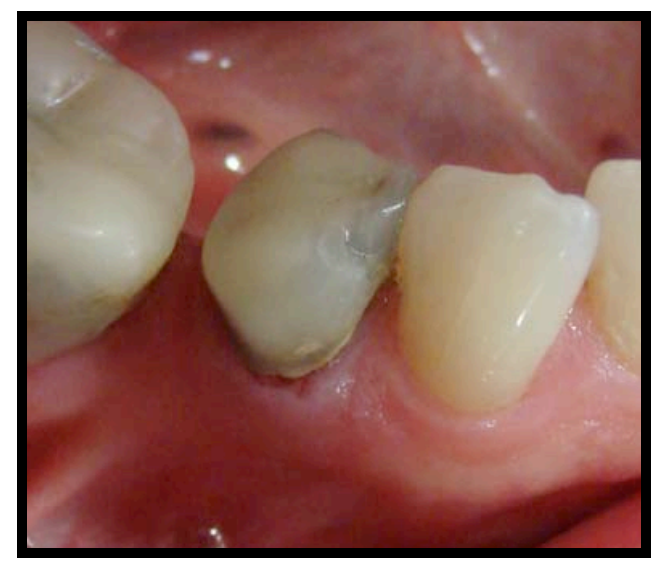

Figura 1: aspecto clínico inicial

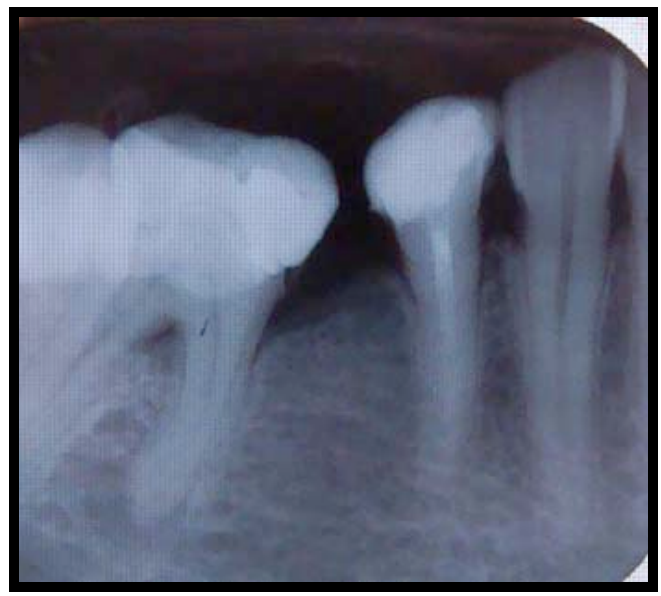

Figura 2: aspecto radiográfico inicial

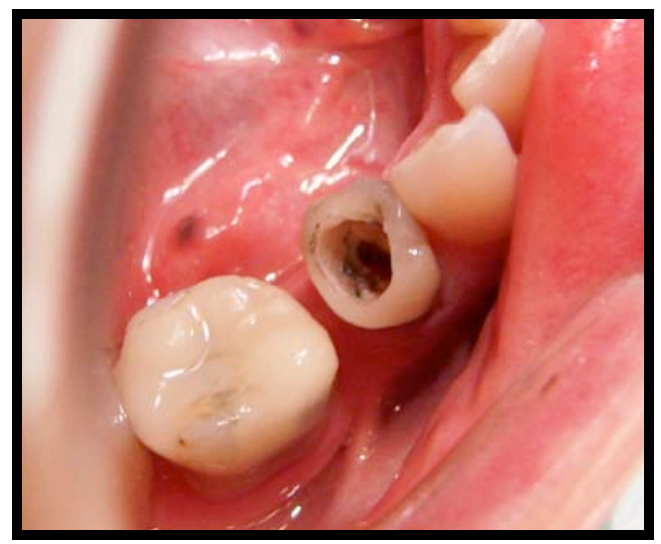

Figura 3: canal desobturado pronto para cimentação de pino pré-fabricado 


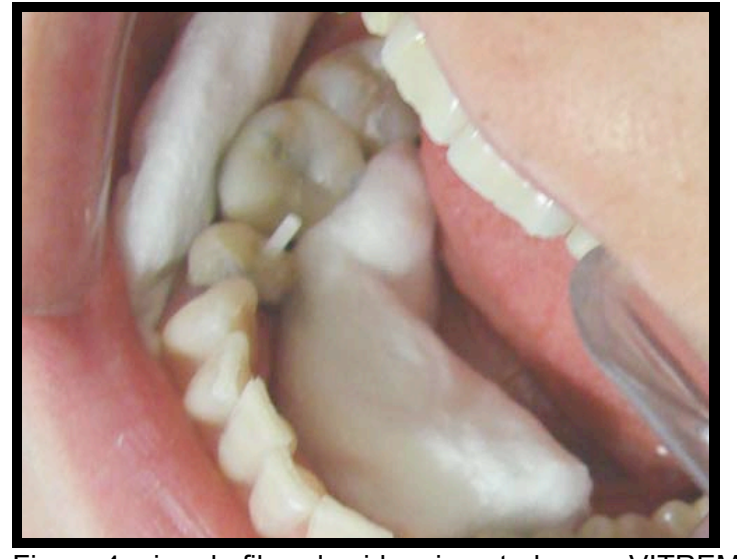

Figura 4: pino de fibra de vidro cimentado com VITREMER.

Foi então realizado o preparo para coroa total com ponta diamantada tronco cônica $n^{\circ} 2135$ (KGSorensen, Barueri, SP, Brasil), removendo todo resíduo de resina composta antiga, deixando apenas o ionômero de vidro como núcleo de preenchimento para a restauração (Figura 5).

$\mathrm{Na}$ seqüência, procedeu-se isolamento absoluto (Figura 6), condicionamento com ácido fosfórico a 37\% (FGM, Joinville, SC, Brasil), e aplicação do adesivo Single Bond 2 (3MESPE, St Paul, MN, USA) para o tratamento adesivo superficial, permitindo o início do procedimento de acomodação da resina composta.

Optou-se pela utilização de matrizes pré-fabricadas (TDV, Pomerode, SC, Brasil) para confecção da coroa direta de resina composta. A resina composta escolhida foi microhíbrida OPALLIS (FGM, Joinville, SC, Brasil).

Após prova e adaptação da matriz de Ableh (figura 7), esta foi preenchida com o compósito resinoso e novamente adaptada ao dente preparado. Removeu-se o excesso de material extravasado (Figura 8) e polimerizou-se por longo período, aproximadamente 60 segundos em cada face do dente (Figura 9).

Removeu-se a matriz com lâmina de bisturi №11 e com ponta diamantada $\mathrm{n}^{\circ}$ 2135F (KGSorensen, Barueri, SP, Brasil), refrigerada em alta rotação, remove-se os excessos de resina composta e realiza-se o ajuste oclusal (Figuras 9 e 10). O polimento final com discos Sof-Lex em baixa rotação (3MESPE, St Paul, MN, USA) foi realizado na consulta seguinte até obtenção da lisura superficial. Nas figuras 11a e 11b observa-se o aspecto final da restauração, com restabelecimento da forma e do ponto de contato.



Figura 5: preparo para coroa total, eliminando o remanescente da restauração antiga, mantendo o núcleo de preenchimento.



Figura 6: isolamento absoluto do campo operatório



Figura 7: prova da matriz de Ableh

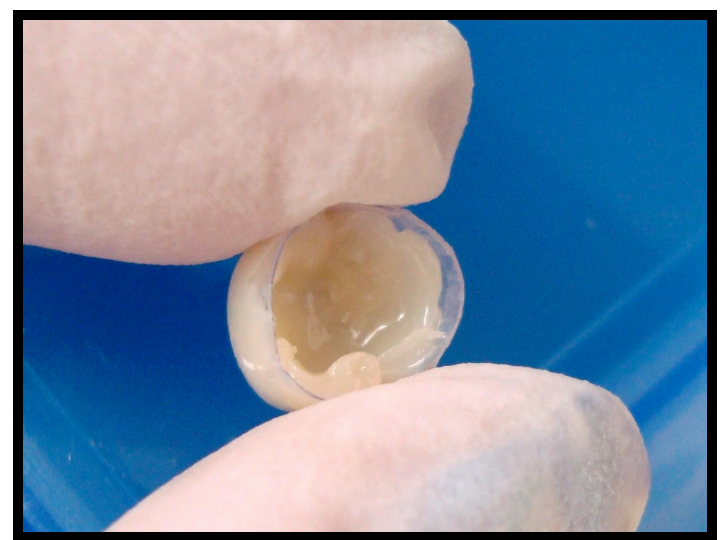

Figura 8: preenchimento com compósito 


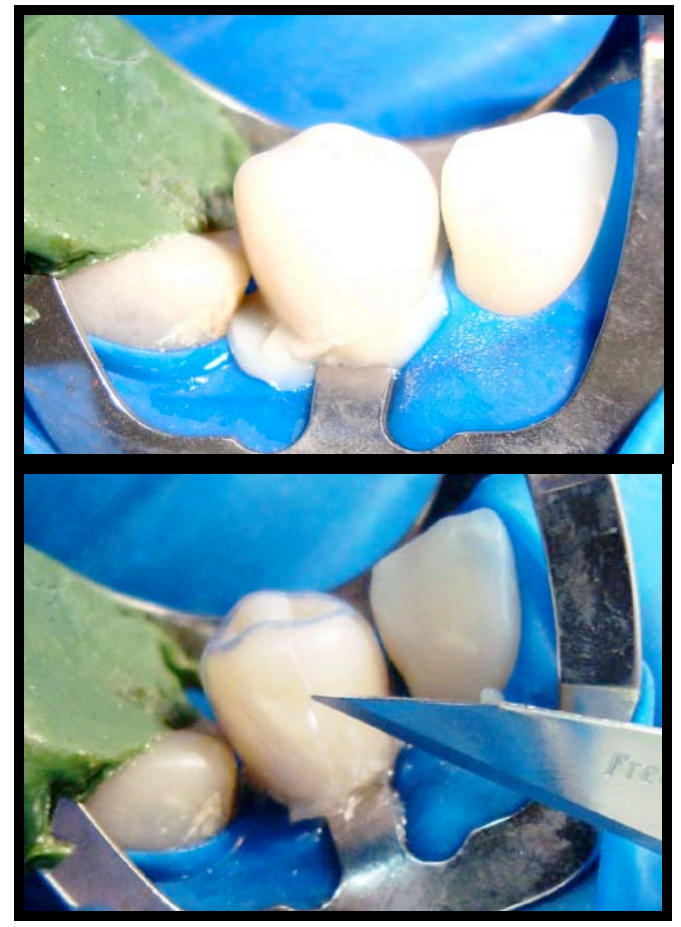

Figura 9: Inserção da matriz em posição com a resina composta; recorte e deslocamento da matriz com lamina de bisturi

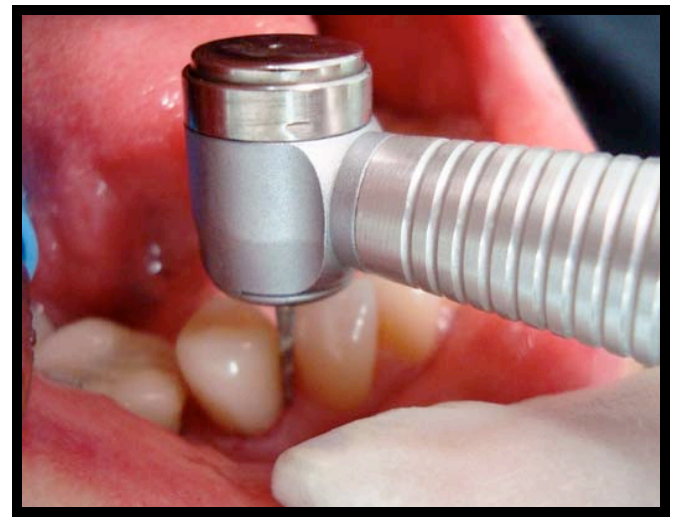

Figura 10: remoção de excesso com ponta diamantada FF.

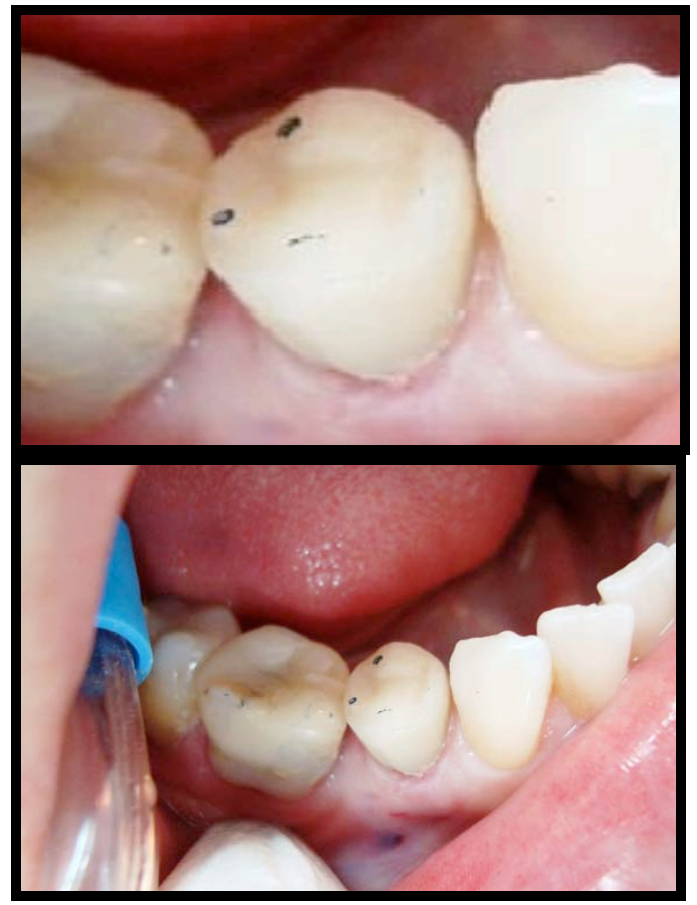

Figura 11a: aspecto final.

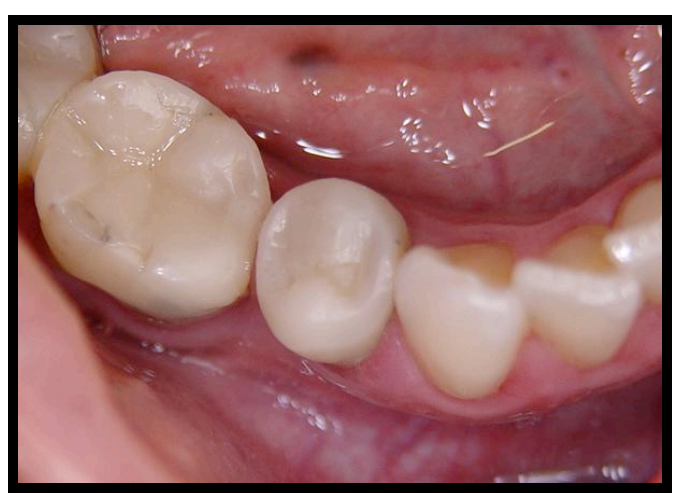

Figura 11b: aspecto final após polimento. 


\section{Relato de caso clínico 2}

Paciente sexo feminino, 45 anos, leucoderma, compareceu à clínica odontológica com dente 12 tratado endodonticamente, parcialmente desobturado e provisório de resina acrílica cimentado temporariamente (Figura 12). Após isolamento absoluto (Figura 13), foi realizada seleção do pino estético de fibra de vidro $n^{\circ} 3$ (WHITE POST DC, FGM, Joinville, SC, Brasil), por melhor se adaptar à luz do canal, deixando um espaço menor a ser preenchido pelo ionômero restaurador.

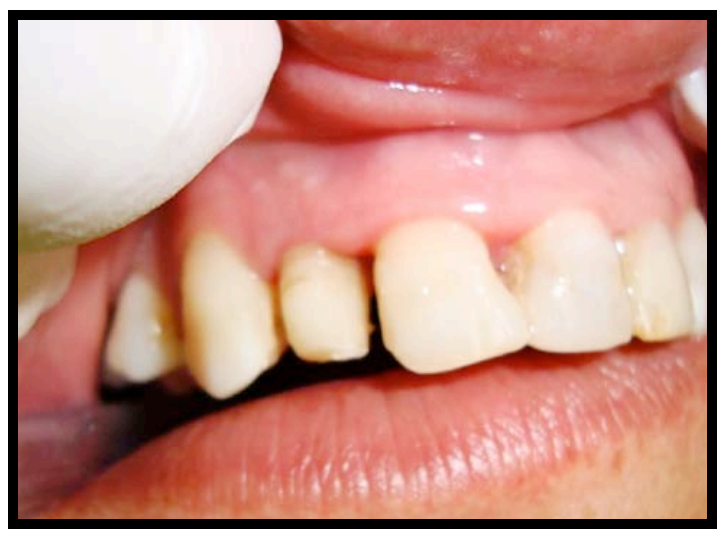

Figura 12: aspecto inicial do elemento dental 12

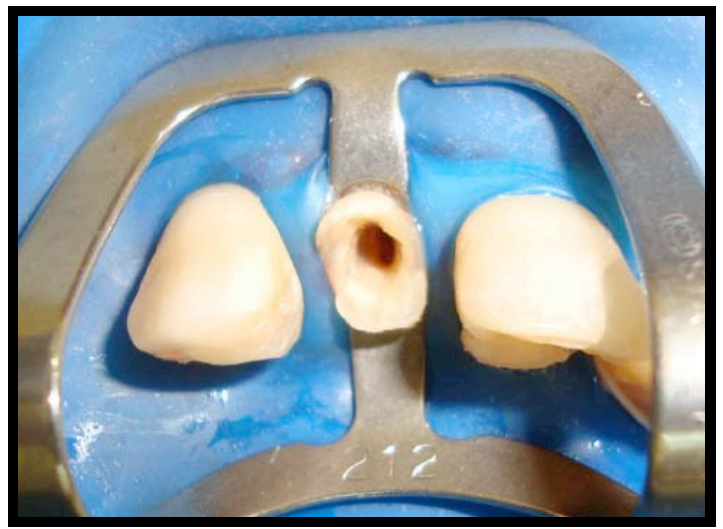

Figura 13: Isolamento absoluto, remoção da restauração deficiente e desobturação endodontica

Com o conduto devidamente preparado, foi feita a inserção do cimento de ionômero de vidro VITREMER com auxílio de broca lentulo (Dentsply Maillefer, Petrópolis, RJ, Brasil) e posicionamento do pino no canal, seguido da fotopolimerização por 60 segundos com fotopolimerizador Optilight LD III (GNATUS, Ribeirão Preto, SP, Brasil) (Figura 14).

Após remoção dos excessos, realizou-se condicionamento ácido do remanescente dentário com acido fosfórico $37 \%$ por 15 segundos, lavagem com água e secagem vigorosa, aplicação do adesivo Single Bond 2 (3MESPE, St Paul, MN, USA), polimerização e início do procedimento restaurador direto com resina composta.

Foi utilizada uma matriz de poliéster para auxiliar na confecção da face palatina do dente 12 (Figura 15). A resina utilizada foi Opallis (FGM, Joinville, SC, Brasil) Microhíbrida na cor A2 de esmalte.

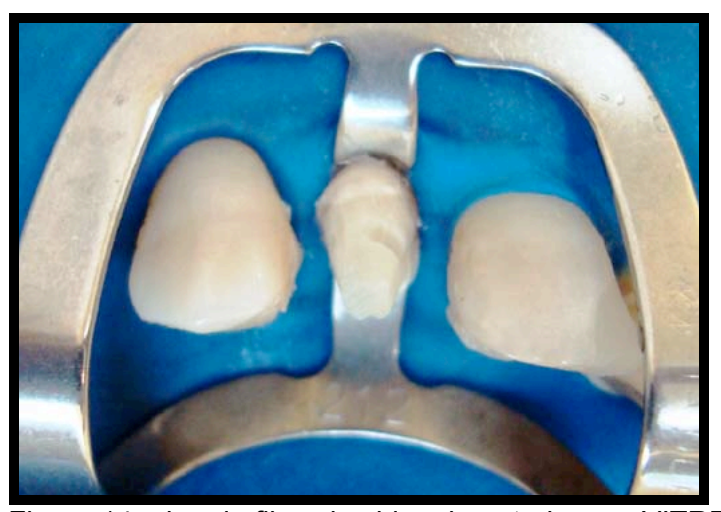

Figura 14: pino de fibra de vidro cimentado com VITREME

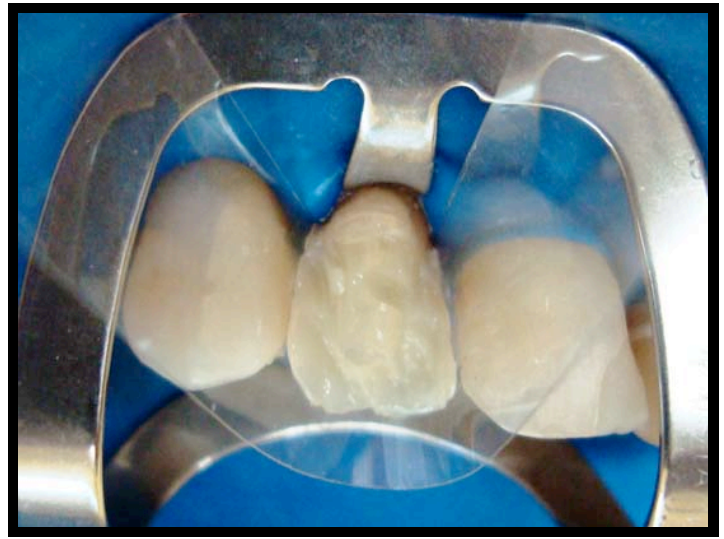

Figura 15: início da inserção da resina composta

Inserindo a resina incrementalmente, usou-se a resina A2 para dentina na extensão do corpo do dente, e após, outra camada de A2 esmalte na vestibular, concluindo o processo de escultura (Figura 16). Realizou-se a fotopolimerização completa com Optilight LD III (GNATUS, Ribeirão Preto, SP, Brasil) por 40 segundos cada incremento. Realizou-se o acabamento e ajuste oclusal com ponta diamantada 2135F e 3118F (KGSorensen, Barueri, SP, Brasil) na superfície palatina, obedecendo a orientação determinada pela marcação de papel carbono, seguindo preceitos de oclusão mutuamente protegida, e polimento com pontas diamantadas FF (KGSorensen, Barueri, SP, Brasil) e discos de Sof-Lex de forma seqüencial tanto em vestibular quanto em palatina, de forma a obter uma superfície lisa, polida, com brilho próximo ao natural (3MESPE, St Paul, MN, USA) (Figura 17). Na superfície proximal, foi realizado polimento com tira de lixas de poliéster divididas ao meio em seu longo eixo, obedecendo a seqüência de granulometria da lixa. 


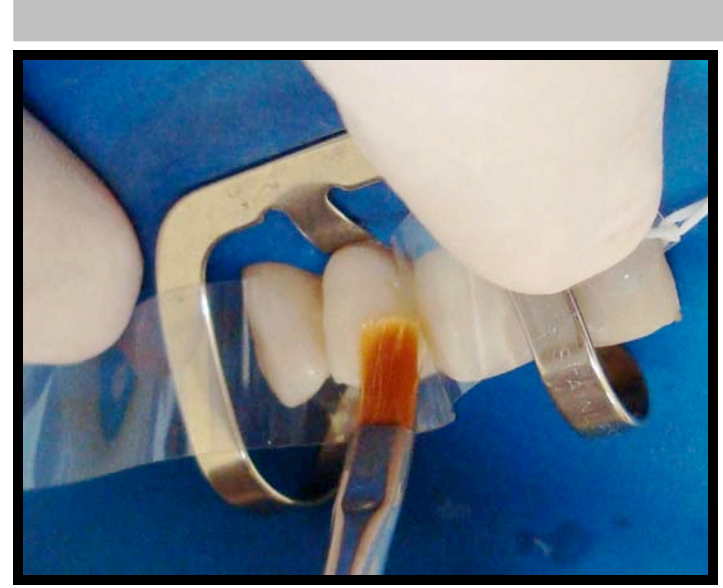

Figura 16: regularização com pincéis da última camada de resina composta

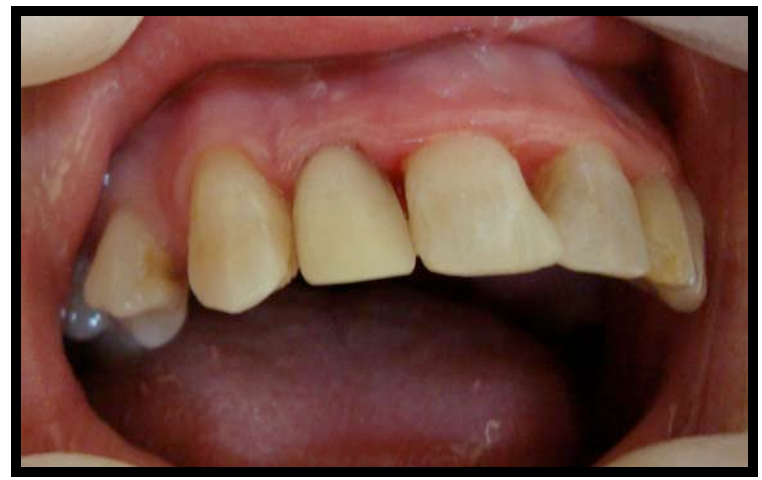

Figura 17: Aspecto no final do procedimento restaurador

\section{Considerações Finais}

De acordo com os artigos revisados percebe-se que houve um aumento no uso dos materiais resinosos principalmente em grandes restaurações de dentes posteriores e anteriores, principalmente associados a pinos intracanais pré-fabricados. As características dos adesivos e resinas e a técnica operatória também exercem um papel importante na durabilidade da restauração.

As resinas compostas foram introduzidas em 1963 e as pesquisas estavam apenas começando, indicando falhas que se deviam freqüentemente a causas como necrose pulpar, sensibilidade pós-operatória, cáries secundárias e fraturas. Hoje em dia, esses materiais evoluíram a ponto de durarem até 10 anos na forma de restaurações, com índice de sucesso ao redor dos 90\% (VAN DIJKEN; PALLESEN, 2011a; VAN DIJKEN; PALLESEN, 2011b; MONTEIRO et al., 2010; SHI et al., 2010). Coroas metalocerâmicas têm aproximadamante 10 a 20 anos de longevidade (ELIASSON;, ARNELUND; JOHANSSON, 2007; NÄPÄNKANGAS; SALONEN; RAUSTIA, 1997; ANUSAVICE; KAKAR; FERREE, 2007) e restaurações de cerâmica pura vem apresentando resultados bastante animadores em pesquisas clínicas, embasando cientificamente um futuro bastante promissor desses materiais (GROTEN; HUTTIG, 2010; FEDERLIN; HILLER; SCHMALZ, 2010; BEUER et al, , 2010; MANHART et al., 2004).

No presente trabalho, as resinas compostas utilizadas foram as microhíbridas por serem resinas com maior quantidade de carga, o que aumenta consideravelmente a resistência, podendo ser indicado para amplas reconstruções e restaurações em dentes posteriores (DA ROSA RODOLPHO et al., 2006; DELIPERI, 2008). O tamanho médio das partículas é de ordem micrométrica, proporcionando um alto polimento, além de ser suficiente para proporcionar uma boa resistência. Os resultados são resinas compostas com boas propriedades físicas e estéticas (SANTOS JUNIOR, 2000; CONCEIÇÃO; MASOTTI; HIRATA, 2005).

Algumas propriedades das resinas ainda deixam a desejar em relação a sua aplicação direta, como a estabilidade de cor, o desgaste oclusal e a contração de polimerização, que leva a falhas na integridade marginal, quando comparadas com as restaurações indiretas (DALPINO et al., 2002; MANFIO et al., 2006). Para tentar amenizar a contração, uma das opções é a utilização da técnica incremental, com polimerização lenta e individual, reduzindo desta maneira os pontos de concentração de tensões na superfície adesiva, além da compensação dos sucessivos incrementos na confecção da coroa, o que gera uma restauração final com maior relaxamento estrutural (DELIPERI; BARDWELL, 2002).

A confecção de coroas totais de resina composta indireta serve como uma alternativa as reconstruções diretas e as porcelanas, com o objetivo de promover a confecção do ponto de contato mais facilmente, anatomia mais detalhada, melhor polimento e deixar a contração de polimerização limitada apenas ao agente cimentante resinoso para a obtenção de uma restauração de maior qualidade e longevidade (MÂNFIO et al., 2006).

Algumas vantagens como conseguir um resultado estético em curto prazo e possuir baixo custo por não ter fase laboratorial, transformaram as coroas totais de resinas compostas numa alternativa viável para situações clínicas específicas, e também como opção para muitos pacientes cuja condição social não permite a confecção de uma restauração de porcelana. A praticidade, já que o número de sessões é menor quando comparado às restaurações indiretas, e a efetividade do tratamento, já que o paciente consegue recuperar sua estética e função, também são vantagens que vem popularizando a técnica tanto entre profissionais quanto pacientes. Uma observação importante é que o sucesso da técnica operatória depende da habilidade do profissional. A restauração é realizada diretamente em boca onde existem desafios que devem ser contornados como controle de umidade, adaptação das margens com o auxílio de matrizes e cunhas, confecção do ponto de contato, o que eleva o tempo clínico necessário ao procedimento.

A influência de fatores como higiene oral e risco a cárie, exercem papel determinante na longevidade da restauração, assim como a idade do paciente. Em pacientes jovens a coroa direta de resina composta poderia ser uma opção prévia à porcelana, em casos de fraturas precoces ou distúrbios de formação, até o completo desenvolvimento do indivíduo. Christensen (2005) diz que restaurações em dentes posteriores variam em preço e longevidade, e sugere que os profissionais sempre informem aos seus pacientes sobre os prós e contras de cada um.

A utilização do Vitremer como material cimentador já vem sendo usado e testado na literatura para sua aplicação na fixação de coroas totais (WILSON; STANKIEWICZ, 1998; YILMAZ et al., 2006; ZORBA; ÖZCAN, 2007). Optamos por utilizá-lo na cimentação de pino por suas características adesivas (BONFANTE et al., 2007), pois isso simplifica o procedimento, eliminando a necessidade de um adesivo que seja ou se torne autopolimerizável, além da complicada etapa de condicionamento ácido do conduto e sua lavagem; e também pois cimentos ionomericos modificados por resina tem resistência flexural semelhante aos resinosos (SASKALAUSKAITE; TAM; MCCOMB et al., 2008). A característica de presa Trial do Vitremer, propriedade única desse material, permite que haja uma cura homogênea ao longo do material no interior do conduto, o que não ocorre com os cimentos resinosos (SIGEMORI et al., 2005; ROBERTS; BERZINS; CHARLTON, 2009). Esse conjunto de evidências exemplificam a literatura que embasa nossa indicação, o que não nos detém na indicação de um cimento resinoso, porém, no 
caso específico, foi usado o cimento ionomérico modificado por resina.

A técnica proposta neste artigo teve como objetivo a recuperação da estética e função dos elementos dentais, através de um procedimento alternativo, de baixo custo, e rápida elaboração, que conseguiu suprir as necessidades do paciente (TAMBELLINI; RAZABONI, 1995; MARTURELLI et al., 2007), mostrando ser uma boa alternativa de tratamento concordando com a literatura existente no que diz respeito ao excelente potencial restaurador das resinas compostas atualmente, quando criteriosamente empregadas, juntamente com um planejamento integral para o tratamento e manutenção do paciente (CAVALCANTI; SANTOS; GUEDES-PINTO, 2002; CHRISTENSEN, 2005; DELIPERI, 2008).

\section{Agradecimentos}

Os casos aqui relatados foram realizados pelas alunas da especialização em Dentística SOBRACID/ULBRA, Pozzatto ANGONESE e Stella MENEGHETTI.

\section{Referências}

ALBUQUERQUE, R. C. et al. Síndrome do dente gretado. Correio ABO, Belo Horizonte, v. 1, p. 20 - 21, out. 2005.

ANUSAVICE, K.J.; KAKAR, K.; FERREE, N. Which mechanical and physical testing methods are relevant for predicting the clinical performance of ceramic-based dental prostheses? Clin. Oral Implants Res., Copenhagen, v. 18 Suppl. 3, p. 218-231, June 2007.

BEUER, F. Prospective study of zirconia-based restorations: 3-year clinical results. Quintessence Int., Berlin, v. 41, no. 8, p.631-637, Sept. 2010.

BONFANTE, G. et al. Tensile bond strength of glass fiber posts luted with different cements. Braz. Oral Res., São Paulo, v. 21, no. 2, p. 159-164, Apr./June 2007.

BOWEN, R.L. Properties of a silica-reinforced polymer for dental restorations. J. Am. Dent. Assoc., Chicago, v. 66, p. 57-64, Jan. 1963.

BRUNTON, P.A. et al. Fracture resistance of teeth restored with onlays of three contemporary tooth-colored resin-bonded restorative materials. J. Prosthet. Dent., St. Louis, v. 82, no. 2, p. 167-171, Aug. 1999.

BUONOCORE, M.G. A simple method of increasing the adhesion of acrylic filling materials to enamel surfaces. J. Dent. Res., Chicago, v. 34, no. 6, p. 849-853, Dec. 1955.

BUSATO, A.L.S. et al. Materiais restauradores e técnicas contemporâneas - separando os fatos da ficção. In: CARDOSO, R.J.A.; GONÇALVES, E.A.N. Dentística, laser. São Paulo: Artes Médicas: APCD, 2001. Cap. 18, p. 315-336.

BUSATO, A.L.S.; HERNANDEZ, P.A.G.; MACEDO, R.P. Dentística: restaurações estéticas. São Paulo: Artes Médicas, 2002.
CAVALCANTI, A.L.; SANTOS, E.M.; GUEDES-PINTO, A.C. Reabilitação bucal em casos de amelogênese imperfeita: relato de caso. Rev. Paulista Odontol., São Paulo, v. 24, no. 3, p. 9-14, 2002.

CHEUNG, W. A review of the management of endodontically treated teeth: post, core and the final restoration. J. Am. Dent. Assoc., Chicago, v. 136, no. 5, p. 611-619, May 2005.

CHRISTENSEN, G.J. Restoring a single anterior tooth: Solutions to a dental dillema. J. Am. Dent. Assoc., Chicago, v. 135, no. 12, p. 17251727, Dec. 2004.

CHRISTENSEN, G.J. Longevity of posterior tooth dental restorations. J. Am. Dent. Assoc., Chicago, v. 136, no. 2, p. 201-203, Feb. 2005.

CHRISTENSEN, G.J. When is a full-crown restoration indicated? J. Am. Dent. Assoc., Chicago, v. 138, no. 1, p. 101-103, Jan. 2007.

CONCEIÇÃO, E.N.; MASOTTI, A.; HIRATA, R. Reproduzindo função e estética com compósitos diretos e indiretos em dentes posteriores. In: CONCEIÇÃO, E.N. Restaurações estéticas: compósitos, cerâmicas e implantes. Porto Alegre: Artmed, 2005. Cap. 5, p. 102143.

DALPINO, P.H.P. et al. Fracture resistance of teeth directly and indirectly restored with composite resin and indirectly restored with ceramic materials. Am. J. Dent., San Antonio, v. 15, no. 6, p. 389394, Dec. 2002.

DA ROSA RODOLPHO, P.A. A clinical evaluation of posterior composite restorations: 17-year findings. J. Dent., Kidlington, v. 34, no.7, p. 427-435, Aug. 2006.

DELIPERI, S. Direct fiber-reinforced composite restoration in an endodontically-treated molar: a three-year case report. Oper. Dent., Seattle, v. 33, no. 2, p. 209-214, Mar./Apr. 2008.

DELIPERI, S.; BARDWELL, D. N. Um método alternativo para reduzir a contração de polimerização nas restaurações posteriores em resina composta. Rev. Jada Brasil, [S.I.], v. 5, n. 5, out. 2002. Cod. A07.

ELIASSON, A.; ARNELUND, C.F.; JOHANSSON, A. A clinical evaluation of cobalt-chromium metal-ceramic fixed partial dentures and crowns: a three- to seven-year retrospective study. J. Prosthet. Dent., St. Louis, v. 98, no. 1, p. 6-16, July 2007.

FEDERLIN, M.; HILLER, K.A.; SCHMALZ, G. Controlled, prospective clinical split-mouth study of cast gold vs. ceramic partial crowns: 5.5 year results. Am. J. Dent., San Antonio, v. 23, no. 3, p. 161-167, June 2010.

GROTEN, M.; HUTTIG, F. The performance of zirconium dioxide crowns: a clinical follow-up. Int. J. Prosthodont., Lombard, III., v. 23, no. 5, p. 429-431, Sept./Oct. 2010.

KRIEGER, LÉO. ABOPREV: promoção de saúde bucal. São Paulo: Artes Médicas, 1997. 475 p.

MAGNE, P.; BELSER, U.C. Porcelain versus composite inlays/onlays: effects of mechanical loads on stress distribution, adhesion, and crown flexure. J. Periodontics Restorative Dent., Chicago, v. 23, no. 6 , p. 543-555, Dec. 2003.

MANFIO, A.P.; BIACCHI, G.R.; GUIMARÃES, M.B.; HABEKOSTE, F.M. Coroa total de resina composta: procedimento alternativo na 
reconstrução do elemento dental. RGO, Porto Alegre, v. 54, no. 1, p. 27-30, 2006

MANHART, J. et al. Buonocore Memorial Lecture. Review of the clinical survival of direct and indirect restorations in posterior teeth of the permanent dentition. Oper. Dent., Seattle, v. 29, no. 5, p. 481508, Sept./Oct. 2004.

MARTURELLI, R. et al. Alternativa estética para reconstrução de dentes anteriores fraturados. Stomatos, Canoas, v. 13 , no. $25, \mathrm{p}$. 123-130, jul./dez. 2007.

MONTEIRO, P.M. et al. Two-year clinical evaluation of packable and nanostructured resin-based composites placed with two techniques. J. Am. Dent. Assoc., Chicago, v. 141, no. 3, p. 319-329, Mar. 2010.

NAKABAYASHI, N.; KOJIMA, K.; MASUHARA, E. The promotion of adhesion by the infiltration of monomers into tooth substrates. $\mathbf{J}$.

Biomed. Mater. Res., Hoboken, N.J., v. 16, no. 3, p. 265-273, May 1982.

NÄPÄNKANGAS, R.; SALONEN, M.A.; RAUSTIA, A.M. A 10-year follow-up study of fixed metal ceramic prosthodontics. J. Oral Rehabil., Oxford, v. 24, no. 10, p. 713-717, Oct. 1997.

OPDAM, N.J.M. et al. Seven-year clinical evaluation of painful cracked teeth restored with a direct composite restoration. J. Endod., Chicago, v. 34, no. 7, p. 808-811, July 2008.

PINTO, V.G. Saúde bucal coletiva. 4. ed. São Paulo: Liv. Santos, 2000. $541 \mathrm{p}$.

ROBERTS, H.W.; BERZINS, D.W.; CHARLTON, D.G. Hardness of three resin-modified glass-ionomer restorative materials as a function of depth and time. J. Esthet. Restor. Dent., London, v. 21 no. 4, p. 262-272, 2009

SANTOS JUNIOR, G.C. A evolução da resina composta. Rev. Fac. Odontol. Univ. Fed. Bahia, Salvador, v. 20, p. 29-33, jan./jun. 2000.

SASKALAUSKAITE, E.; TAM, L.E.; MCCOMB, D. Flexural strength, elastic modulus, and $\mathrm{pH}$ profile of self-etch resin luting cements. J. Prosthodont., Copenhagen, v. 17, no. 4, p. 262-268, June 2008.

$\mathrm{SHI}$, L. et al. Evaluation of packable and conventional hybrid resin composites in Class I restorations: three-year results of a randomized, double-blind and controlled clinical trial. Oper. Dent., Seattle, v. 35, no. 1, p. 11-19, Jan./Feb. 2010.

SIGEMORI, R.M. et al. Curing depth of a resin-modified glass ionomer and two resin-based luting agents. Oper. Dent., Seattle, v. 30, no. 2, p. 185-189, Mar./Apr. 2005

SPOHR, A.M.; CONCEIÇÃO, E.N.; Fundamentos dos sistemas cerâmicos. In: CONCEIÇÃO, E.N. Restaurações estéticas: compósitos, cerâmicas e implantes. Porto Alegre: Artmed, 2005. Cap. 8, p. 198-217.

TAMBELLINI, M.M.; RAZABONI, A.M. Dente precocemente indicado para prótese: restauração conservativa. RGO, Porto Alegre, v. 43, no. 5, p. 267-270, 1995.
THYLSTRUP, A. e FEJERSKOV, O. Cariologia Clínica. 2. ed. São Paulo: Santos,

2001.

VAN DIJKEN, J.W.; PALLESEN, U. Clinical performance of a hybrid resin composite with and without an intermediate layer of flowable resin composite: a 7-year evaluation. Dent. Mater., Copenhagen, v. 27 , no. 2, p. 150-156, Feb. 2011a.

VAN DIJKEN, J.W.; PALLESEN, U. Four-year clinical evaluation of Class II nano-hybrid resin composite restorations bonded with a onestep self-etch and a two-step etch-and-rinse adhesive. J. Dent., Kidlington, v. 39, no. 1, p. 16-25, Jan. 2011b.

WILSON, P.R.; STANKIEWICZ, N.R. Effect of cement space and delayed placement on the seating of crowns luted with Vitremer, Fuji Duet and Dyract Cem. Am. J. Dent., San Antonio, v. 11, no. 5, p. 240-244, Oct. 1998

YILMAZ, Y. et al. Evaluation of stainless steel crowns cemented with glass-ionomer and resin-modified glass-ionomer luting cements. Am. J. Dent., San Antonio, v. 19, no. 2, p. 106-110, Apr. 2006.

ZORBA, Y.O.; ÖZCAN, E. Reattachment of Coronal Fragment Using Fiber-Reinforced Post: a case report. Eur. J. Dent., Ankara, v. 1, no. 3, p. 174-178, July 2007. 